\title{
Designing Interactive Technology for Skateboarding
}

\author{
Sebastiaan Pijnappel Florian 'Floyd' Mueller \\ Exertion Games Lab \\ RMIT University \\ Melbourne, Australia \\ \{sebastiaan, floyd\}@exertiongameslab.org
}

\begin{abstract}
Interactive technology is increasingly used to support physical activities. However, there is limited knowledge about how interactive technology should be designed to support trick-focused experiences such as skateboarding. We developed Copy Paste Skate, a novel multimodal feedback system, and studied its use by 21 avid skateboarders to explore the design of interactive technology for skateboarding. Based on observations and interviews we articulate two key design dimensions that highlight how designing for skateboarding means supporting execution quality of tricks as well as supporting the trick originality. We also present 4 design strategies to help designers support both dimensions in one integrated design. Our work extends designers' knowledge about how to design interactive technology for skateboarding, ultimately extending our understanding of how interactive technology can support people being physically active.
\end{abstract}

\section{Author Keywords}

Skateboarding; exertion games; trick-focused sports.

\section{ACM Classification Keywords}

H5.m. Information interfaces and presentation (e.g., HCI):

Miscellaneous.

\section{INTRODUCTION}

Interactive technology has increasingly been examined to support the sports experience. For example, research has shown that interactive technology can help professional athletes improve their speed skating [21], rowing [4] and swimming techniques [9] in order to reach the finish line faster. Popular commercial systems such as Nike+ [12] and Runkeeper aim to support joggers by providing feedback that is meant to motivate running faster and more often. However, there is limited knowledge about how interactive technology might be able to support the experience of trickfocused sports, such as skateboarding, freestyle snowboarding, BMX, surfing or freestyle motocross. These sports revolve around the experience of carrying out skillful aesthetic maneuvers, i.e. tricks.

Permission to make digital or hard copies of all or part of this work for personal or classroom use is granted without fee provided that copies are not made or distributed for profit or commercial advantage and that copies bear this notice and the full citation on the first page. To copy otherwise, or republish, to post on servers or to redistribute to lists, requires prior specific permission and/or a fee.

TEI 2014, Feb 16-19, 2014, Munich, Germany.

Copyright 2014 ACM 978-1-4503-2635-3/14/02 ...\$15.00.

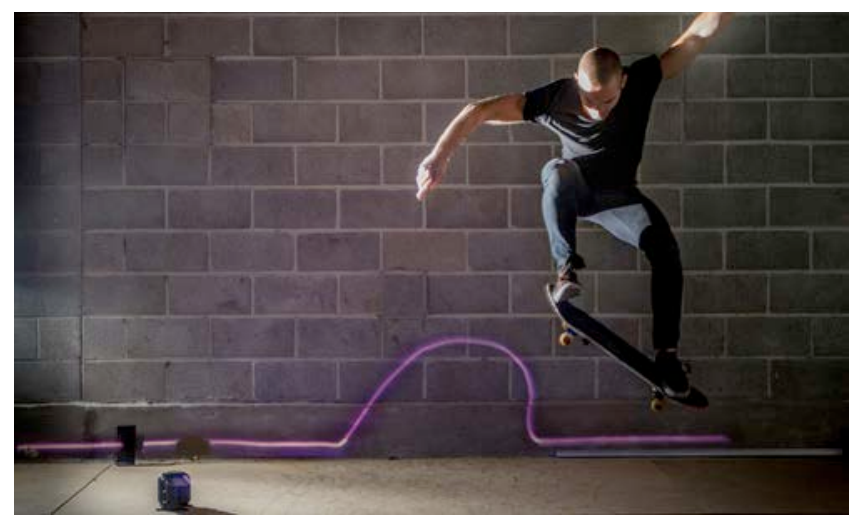

Figure 1. Copy Paste Skate

Considering that we have previous experience with skateboarding [15], we begin our investigation into trickfocused sports with a focus on skateboarding. We have designed and studied the use of a novel multimodal system called Copy Paste Skate, which aims to support the trick experience of skateboarding. Based on observations and interviews with 21 avid skateboarders, we reveal two key design dimensions. These highlight that designing for skateboarding means supporting both trick-execution quality as well as supporting the originality of tricks. We also present 4 design strategies as to how designers can achieve this. We thereby aim to aid designers in the creation of interactive technologies for skateboarding, and to inspire practitioners and researchers to consider opportunities of interactive technology for trick-focused sports in general. With this work, we ultimately hope to extend our understanding of how interactive technology can support people being physically active.

\section{RELATED WORK}

Work on interactive systems for skateboarding is sparse, yet valuable. For example, Tilt n' Roll $[1,16]$ displays visual feedback on a skater's smartphone directly after trick attempts to help learning tricks. This shows us that simple technologies (accelerometers) can be used to capture skateboarding data. However, studies with the system suggested that shortcomings in the precision and resolution of the visual feedback limited the value it can have for skaters [16]. Langlotz et al. [8] discuss a smartphone app that offers more detailed visual feedback by allowing skaters to layer and compare videos of trick attempts to support trick learning. Skataviz [20] also explores layering of information types, overlaying accelerometer data over 
video recordings, which can then be reviewed on a computer screen. Both systems hint at interesting opportunities for visual feedback to enhance a human's vision capabilities to support the skating experience. We took this on board in our design. However, as with Tilt n' Roll, the quality of the visual feedback in both systems is limited (e.g. jagged, interrupted graphs in Skataviz). Tests with Langlotz' system indicated visual limitations harmed the efficacy of the system [8]. In sum, all three systems suggest there might be benefit in providing rich visual (and possibly other modalities of) feedback for skateboarders.

Higher quality feedback has been incorporated in two skateboarding installations built for public events. The Skate Sonic system [14] translates movements of skaters into a pleasing "sonic landscape", adding an aesthetic level to the otherwise mostly functional aural feedback in skateboarding. The Tron Ramp [6] is a skateboarding ramp augmented with elaborate visual effects projected around skaters according to their movements. We note that both these systems aim to support the skateboarding experience in a way that leaves the original skateboarding movements unaffected, yet they provide digital feedback in response to these movements. Inspired by these systems we decided to also focus on supporting the skateboarding experience with digital feedback, while celebrating the core activity that skaters are interested in: attempting tricks. Unfortunately, no research has been conducted around these systems. Our investigations extend this work by providing evidence as to if and how interactive technology can support the skateboarding experience.

In order to better understand the skateboarding experience we look at work by Seifert and Hedderson [18], who tell us that emotions which are key to the experience of attempting tricks occur mostly right after the attempt. In response, we also focus on the emotional component of the experience, with an emphasis on the period right after an attempt.

Skateboarders value the use of technology such as video recording to capture, relive and share their experiences [3]. However, capturing and sharing video is time consuming, with the sharing usually taking place only after, not during, the skateboarding session. Virtual computer games such as Tony Hawk's Pro Skater [23] show examples of more immediate feedback to support the skating experience, such as instant replay or color tagging of obstacles. Our work draws on the idea of immediate feedback and the use of technology to review parts of the skateboarding experience immediately after trick attempts.

In sum, skateboarding theory and existing systems suggest an opportunity for interactive technology to support the skateboarding experience. However, there is little systematic understanding of how technology can and should be designed to support this experience. Our earlier work [15], an autoethnographical study using manually triggered low-fidelity systems, offered insights into what designers should be mindful of when designing for skateboarding. However, this work did not yet articulate ways in which interactive technology can affect skateboarding, nor did it recommend design decisions to facilitate those. By building a novel, fully automated skateboarding system (called Copy Paste Skate) and studying how skaters use it, we aim to provide this missing knowledge. Specifically, we will identify ways in which interactive technology can affect the skateboarding experience, dissect these experience aspects to articulate how system features can support them, and provide design strategies.

\section{THE COPY PASTE SKATE SYSTEM}

Our approach to exploring interactive technology and skateboarding was to design and study the use of a novel interactive system: Copy Paste Skate. We know explain the system's design more fully, structured along three sensory modalities of feedback on tricks: visual, aural and haptic, as proposed by Tholander et al. [22]. The authors cite the example of skaters visually reading their environment, while also feeling its texture through the skateboard and hearing the board land on the floor as a way of getting rich feedback on tricks. Tholander et al. argue that this inherent feedback across these three sensory modalities is key to the skaters' perception of their tricks, which in turn is key to the skateboarding experience.

\section{Visual feedback: Projected skateboard movements}

A still visualization showing the path of movement of the skateboard during the trick is projected onto the wall just behind the skater in life size. Each subsequent visualization is projected on top of the previous one. Old visualizations disappear after 3 attempts.

While skaters often use video recordings to evaluate their performance after a skateboarding session, we opted for static and abstract visuals. Our autoethnography work with a range of early prototypes suggested that static and abstract visuals allow for quick interpretations of a trick whenever skaters desire. In contrast, a video can demand more cognitive attention, which could negatively affect the skateboarding experience [15]. By representing movements as a single line, and omitting the body and environment, we aim to accentuate movement qualities of the board.

The projected skateboard movements are realized through small custom-made infrared emitters that are taped to the bottom of the skateboard. These consisted of 5 LEDs (SFH487) packed in a $15 \times 15 \times 60 \mathrm{~mm}$ housing, weighing under $50 \mathrm{~g}$ in order to not notably affect the skating. An ultrasonic proximity sensor detects the skater approaching the area and triggers an infrared-sensitive camera that captures the movement of the board for 2.5 seconds. The resulting visual is layered on a maximum of 3 previous visuals, each with an opacity of $80 \%$ to expose the previous visuals underneath. Two daisy-chained projectors, needed to achieve life-size projections, then project the resulting image (Figure 1). 


\section{Aural feedback: Environment}

Directional microphones (Senheiser ME 66) capture the audio produced by the trick attempt for 2.5 seconds, triggered by the same proximity sensor as the visual feedback. This audio is then replayed at $0.5 \mathrm{x}$ speed and $1.5 \mathrm{x}$ volume through large high-quality speakers.

The choice to use the original audio of skateboarding came from our aim to capitalize on the rich information this audio possesses for skaters [22]. Our own previous work [15] indicated it can be hard to pick up on this information, as tricks, and thus the produced audio, only last very shortly. Hence Copy Paste Skate uses slowed-down audio.

Our earlier work [15] also told us that a skater's ability to move freely and continuously through a space forms an important part of the skating experience, and that technology should not interfere with this. In Copy Paste Skate the high volume allows the sound to be heard clearly throughout the space, meaning the skater can move about without having to focus on or be in a specific spot to hear the produced sound.

\section{Haptic feedback: Floor vibrations}

The skateboarding area is outfitted with roughly $60 \mathrm{~m}^{2}$ of medium-density fibreboards (MDF) (Figure 2). These boards are similar to those often used as flooring in skateparks. Right after a trick attempt, these floorboards on which the skater lands and rolls away are vibrated strongly in the rhythm of the motions of the board during the attempt. This offers a replay of the haptic experience felt during a trick attempt. We use a specialized low-frequency audio transducer to produce strong vibrations [5]. The vibrations are produced based on the audio recording, which peaks in time when the board takes off, lands or slides over an obstacle. More powerful takeoffs or harder landings result in stronger haptic experiences. Vibrations carry from the board up through the body, as is the case with any impacts normally felt during a trick.

The aim was for the vibrations to retain the nuances of impacts and vibrations normally felt during trick attempts (e.g. differences between hard and soft, or neat and sloppy landings). Hence, the vibrations are a continuous rather than a discrete interpretation of the skater's movements, i.e. nuances in intensity and length of the vibrations correspond to nuances in motions of the skater.

Making the technology part of the environment allowed us to avoid size and weight restrictions faced by wearable sports systems. Our audio transducer weighs $10 \mathrm{~kg}$ and produces high-intensity vibrations. We felt the high level of sensory stimuli involved in trick-focused sports meant the feedback had to provide a comparable level of stimuli in order to become part of the skating experience. In addition, adding technology to the environment rather than to the board allows skaters' tradition of maintaining the integrity and lo-tech quality of the board [3] to be honored.

\section{STUDY}

We recruited 21 avid skateboarders from the local skateboarding community. All participants were males (skateboarding is a strongly male dominated sport) between 18 and 30 years of age who skate for at least 5 hours a week. Their experience ranged from 3 to 14 years, with all participants at least able to perform basic tricks such as jumping. We felt this demographic approximates a cross section of the local skateboarding community, excluding the below 18 age group. In each group of participants at least two of them were friends, with a third and/or a fourth being a stranger. This mimics a mix of friends and strangers as typically found amongst clusters of skaters in skateparks.

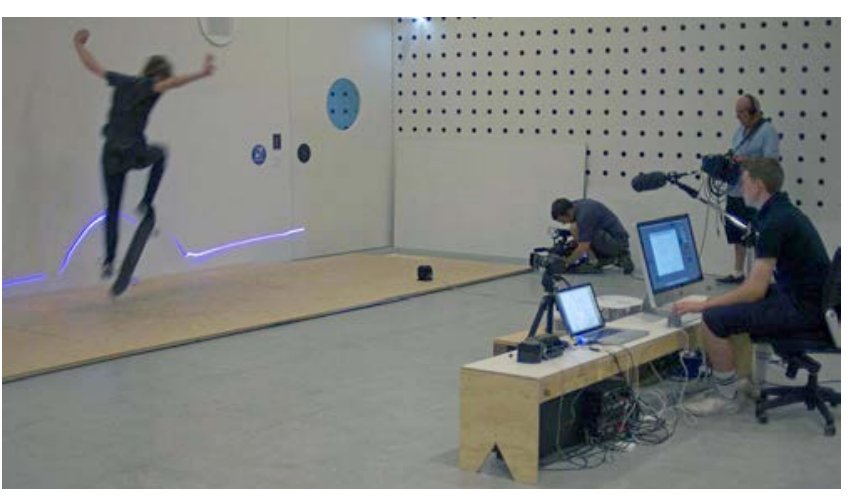

Figure 2. The setup used for our study.

\section{Environment}

We set Copy Paste Skate up in a large indoor space similar to that of an indoor skatepark. However, due to technical limitations, skaters were required to approach the area covered by the system from a specific direction.

\section{Procedure}

Skaters came in to skate with Copy Paste Skate in seven separate sessions. Before switching on the system we asked them to skate freely in the space as they normally would in order to get used to the environment. After 15 minutes we explained Copy Paste Skate and asked them to skate freely using the system, this time for 30 minutes. We stressed that we had no expectations regarding their performance or skill and that they were free to take breaks at any time.

To mimic the freedom to move on to the next obstacle whenever desired, which skaters typically do when skating, we offered two configurations that we swapped several times throughout each session upon skaters' request. The system functioned in the same way for both setups:

- Open space with no obstacle to facilitate "flat ground tricks", which involved maneuvering the board in the air.

- A simple grind-box obstacle placed in the center of the space to facilitate "grinds", which involved sliding or scraping the board over the edge of the obstacle. 


\section{Data Collection and Analysis}

After the skating session, participants were invited to a semi-structured interview. Both the skateboarding sessions and interviews were videotaped, using two cameras from different angles to capture both the big picture as well as facial expressions. The interviews resulted in 23000 words of transcription, which we analyzed by coding and then affinity diagramming to identify key findings.

\section{EXPERIENCING COPY PASTE SKATE}

Our guiding question was: how do skaters engage with Copy Paste Skate? The numbers behind the quotes refer to the participant's group and number. For example, "5.1" means the quote came from skater 1 of the fifth group of skaters. Each finding is numbered (F1-F10) for later referencing.

\section{F1: Doing Tricks for a "Cool" Projected Line}

13 skaters pronounced that they found the projections visually pleasing:

"My favorite would have been the visual cause it really just looks amazing." (5.1)

"It looks a whole lot cooler than some of the other styles and ways that you can look at a trick!" (6.2)

Skaters mentioned several qualities of the line that they liked and that they tried to produce with their tricks. For example, they aimed to create a curvy as opposed to a straight line. The line appeared to facilitate an exploratory approach to the trick experience:

"A straight line is boring, and you just want to see it go all over the place. [...] It was like thinking of what would be the craziest trick to make a weird line. It was fun." (7.1)

The lines' aesthetic qualities seemed to support positive emotions in case of a good trick execution:

"It's like a self-esteem boost cause you landed, look back, it looks sick on that, it sounded good, the pops looks good. Like yeah, that was a good trick!" (6.3)

According to some skaters, the audio did not have this "cool" quality:

"If the audio goes: prrrg prrg prrggg, or prrggg... prgg prgg, or whatever, its not going to make you feel any different about the trick, whereas with the line, you get a cool looking line." (7.4)

\section{F2: New Motivation for Tricks}

8 skaters described how they skated for the projected lines, instead of for the trick itself, where they were less concerned with achieving a certain technical difficulty of tricks (see Figure 3):

"With the system you approached skateboarding a little bit differently. You don't approach it as: I have to land this trick because of its technical difficulty. You approach it as: how can I manipulate my board to create a cool effect? It really opens up an entirely different world." (5.2)
"Tricks you wouldn't normally do, you do here just to kind of find out what they would turn out like." (7.1)

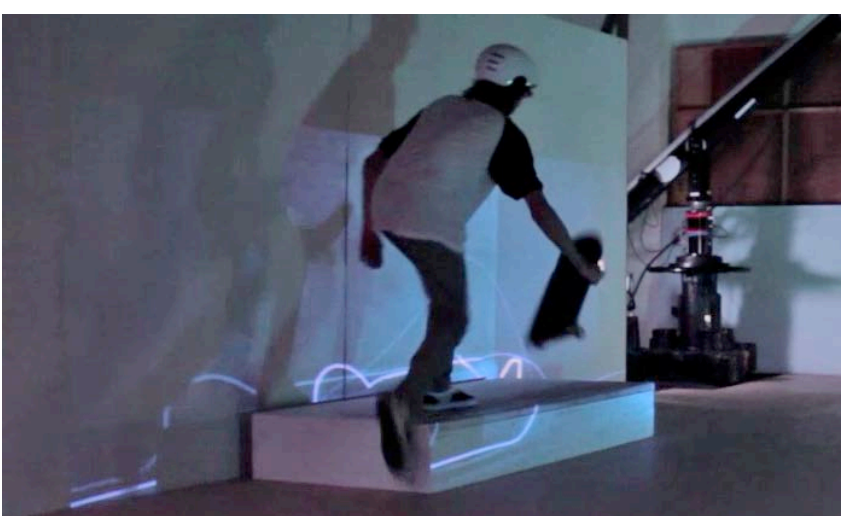

Figure 3. Skater waving the board with his hands to make a curvy line.

\section{F3: Pushing Oneself}

Skaters used the projected line to push themselves to be more creative in selecting tricks:

"It's just the board, it's just the lights. And so, you have to push yourself to be more creative in terms of what tricks you choose." (5.2)

\section{F4: Pride Through Creation}

Another strong theme coming out of the data was how skaters saw the lines as creative creations, and how these creations engendered feelings of pride:

"There's definitely pride in it, I mean, you've created it. A lot of people think that skateboarding is an artistic form, and it's adding another variable to that, like showing skateboarding in a different light. Literally, haha." (3.2) "It's the same way as you feel when you've drawn a picture. It's like an artistic sense of accomplishment. [...] You're actually creating something." (5.2)

\section{F5: Increased Level of Information About the Trick Execution}

Skaters also commented on how the audio and vibrations offered more detailed information about their trick than what they would normally perceive when skating: "Sometimes when you come out of a trick you revert it a little bit when you land and it's not quite as clean. But with the audio you can hear that straight out and go back and do it again clean." (6.2)

"I like the audio, like how it was a bit slower as well so you could, that sort off helped with understanding where you were with the trick." (4.1)

"You can feel the trick you've just done. Like you can tell exactly what's happening in feeling the vibrations." (6.1)

The fact that projections of sequential attempts were layered on top of each other also helped skaters see how their execution had improved:

"Having tricks overlaid one after the other is just really cool, to see how I've improved, even slightly." (4.1) 


\section{F6: Abstracted Representations Supported Focusing on} Specific Trick Aspects

The fact that the projected lines show an abstracted representation of the movement of the board was helping skaters to focus on the trick:

"You can purely focus on the movement. [...] If want to see where your board has been, how high you can get, and the length, so just purely the trick, then this is a much better tool to push yourself to get better than just watching video, cause in the video you're more focused on yourself." (4.1)

However, two skaters also indicated that the level of abstractness limited the informational value:

"This is missing the body. [...] Like it's hard to obtain a lot of information. Like when you do a trick it's like, wow, it looks cool, but it doesn't tell me that much." (1.3)

One skater pointed out that the constant presence of the projections in the space can be distracting: "The actual projection, it sort off is a bit visually distracting. Maybe dull it a little bit.” (6.3)

\section{F7: Gaining Inspiration from Others' Outcome}

An aspect of the system commented on by skaters was the fact that projections of other tricks persisted in the space for others to see. This inspired skaters' own trick attempts: "Seeing someone else's [tricks] gave you inspiration to do something better, cause you could see their results." (7.2) "You base your tricks around what they're doing. If their outcomes look aesthetic or the lines are creative or really cool, then you try to mimic it or be more creative than that." (5.2)

\section{F8: Competitive Comparison with Others' Outcome}

The fact that sequential trick attempts were layered on top of each other inspired competitive play. Two skaters of the first group started a game of who can do the highest jump:

"This was pretty much the first high ollie contest in my life, haha." (1.2)

"We wouldn't have played the game if it wasn't for this [referring to the line]. It kinda brought on a different way of us skateboarding. Cause we could kinda compare each other to do better." (1.3)

\section{F9: Reliving Tricks}

Another strong theme coming out of the data was how the system enabled our skaters to vividly relive the trick after the attempt. Particularly the audio and vibrations were mentioned as contributing to this:

"It's like sort off reliving the trick. [...] It brings the trick to life you know. I think that's what the vibration and the sound does. [...] You were able to reconstruct it in your head more, and relate the sound to the trick." (4.1)

"When you feel the vibration, it's really just a much more lively experience than watching on a screen.” (5.1)
F10: Offering a New Perspective on Execution

Copy Paste Skate revealed new aspects of tricks that skaters were not usually aware of. They said that the system taught them about specific tricks they had performed:

"When I saw the board went like totally vertical [...] I didn't realize how the board went under my feet. [...] It almost graphs your trick, like where it peaks and stuff. It's a new context, a new perspective." (6.2)

'It was interesting to see the length of time I was in the air. And, haha, certainly the impact of coming down [as represented by the vibrations and audio][...] Like you could sort off hear where you popped it, how much did you pop it, so I think that helped me improve." (5.2)

\section{DESIGN DIMENSIONS}

Our findings suggest to us that designing for skateboarding means designing for a wide range of different quality characteristics of the trick experience, and that skateboarders will engage with interactive technology in a way that supports this multi-dimensionality of the trickexperience. In particular, we identify two key dimensions for interactive skateboarding technology to be considered by designers: support for an execution quality-focused trick experience and support for an originality-focused trick experience. We will now discuss each of these. Our findings range from insights into the efficacy of deliberately designed features, to incidental qualities of the system that affected the experience.

It is important to note that the skateboarding experience also encompasses aspects such as performing for a crowd (e.g. in competitions) or the sense of belonging to the skating sub-culture [3]. Although these aspects were touched upon by our participants, we begin our work with a focus on the individual's trick experience, leaving larger contextual issues for future work.

\section{Dimension 1: Support for an Execution Quality-Focused Trick Experience}

This dimension is concerned with the use of interactive technology to support executing tricks as "perfect" as possible, bringing about a skateboarding experience in which skaters try to push themselves to do a trick as high and as neatly as they can. Our skateboarders used certain design features of Copy Paste Skate to support an execution quality-focus.

Layered representations can support comparisons of execution qualities $(F 5+8)$ : Our skateboarders used the fact that projections of sequential trick attempts were layered on top of each other to inspire a drive for execution quality as it allowed the identification of even very small improvements over their previous trick.

Sharing of visuals can afford execution quality comparison $(F 7+8)$ : Skaters used the system's affordance to look at each other's abstract lines, and thus the execution quality of each other's tricks, to try and do a better execution of a trick (e.g. higher) than their peers did. 
Abstract representations can entice skaters to push their execution quality with regard to specific trick aspects (F6): The abstract projections highlighted certain aspects of tricks (e.g. height), which channeled skaters' efforts towards optimizing these aspects. However, as participants also expressed missing other execution quality components that they had hoped to learn about (e.g. body in relation to the board), we can argue that the abstract nature of the representations might have hampered intentions to improve on other trick aspects.

Altered audio can offer new information about execution quality aspects $(F 5+10)$ : The altered audio allowed skaters to gain information about execution quality that would otherwise go unnoticed (such as intensity and timing of takeoff and landing). This was used to improve future attempts.

Vibrations can make mental picture of execution quality more vivid (F9): While the altered audio and abstract visuals provided skaters with information that helped them picture their attempt in their head, being able to feel their takeoffs and landings through the vibrations brought that experience "to life", contributing towards a more vivid mental picture of the trick execution.

The above suggests that athletes can and will use technology to improve their performance, as highlighted before by work on other sports systems [9,21]. Skateboarding theory suggested previously that skateboarders are less concerned with performance variables [18]. In contrast, Copy Paste Skate shows that, when given the opportunity, skateboarders will use interactive technology to support trick execution quality. This fits with self determination theory, which states that humans have an innate drive for competence, particularly in sports [17]. Here we see how technology can facilitate and foster this drive, even in skateboarding.

We can imagine future versions of Copy Paste Skate to support an even higher focus on execution quality. For example, the system could show executions of professional skateboarders to allow participants to compare their own tricks to, or it could allow for the saving and sharing of previous tricks that a skater is particularly proud of.

\section{Dimension 2: Support for an Originality-Focused Trick Experience}

This dimension deals with the extent to which interactive technology supports exploring new and unconventional tricks, bringing about a skateboarding experience that is about originality rather than about doing conventional tricks. Through our study we identified various ways in which Copy Paste Skate supported originality-focused trick experiences.

Aesthetic trick representation can motivate exploration $(F 1+2)$ : Skaters challenged themselves to think beyond conventional tricks to achieve results that were satisfying for them, such as aesthetically pleasing lines. This resulted in skaters exploring alternative ways to perform tricks, even if this meant breaking the (unwritten) rules of skateboarding (e.g. putting the feet on the ground as part of a trick).

Sharing of aesthetic trick representations can fuel desire for originality (F7): The fact that the lines were public, i.e. visible to anyone in the space, further added to the desire to produce satisfying lines, as skaters were inspired by other skaters' lines and discussed these with them to come up with even more original tricks.

Persistent trick representation can afford artistic creation (F4): The fact that the system enabled skaters to create something that lasted beyond the trick facilitated a sense of artistic creation, furthering a feeling of accomplishment.

Abstract trick representation can facilitate the creative process (F3): While video or photos also afford creation through skateboarding tricks, Copy Paste Skate limits the creative output, as it shows only a single line as opposed to a highly realistic depiction of reality. Limitations are believed to foster creativity [10], and Copy Paste Skate suggests that an abstract trick representation can facilitate the creative process of coming up with original tricks.

Different medium of representation can offer new perspectives to support exploration of unconventional tricks (F2): The fact that Copy Paste Skate introduced an unfamiliar representation of the skaters' actions inspired thinking about their tricks from a new perspective. This seemed to allow skaters to reconsider what moves or actions would give them a satisfying result, enabling them to put aside assumptions of which tricks are good or bad and experiment with moves they normally would not do. In creativity theory, introducing different forms of representation is a common method to help question assumptions and come to new ideas, as described in Thinkertoys [10]. Copy Paste Skate highlights an opportunity for designers to use technology to deploy this strategy in skateboarding by representing tricks through new media.

Supporting originality is particularly important in skateboarding, as skateboarding culture highly values creative expression, autonomy and originality [3,11]. Originality contributes to a person's autonomy [17]. However, supporting originality could potentially distract from the essence of the sport: Borden reminds us that skateboarding is about a conversation with the architectural environment [3], while the visuals of Copy Paste Skate could be interpreted as a conversation with the line.

We can imagine future systems improving on Copy Paste Skate's support for originality by finding ways to achieve aesthetic representations not only in the visual domain, but in the aural and haptic domain as well. The perceived absence of these qualities in our system's aural and haptic feedback might have contributed to skaters' tendency to focus on the visual aspect of the system in the interviews. 


\section{DESIGN STRATEGIES}

We now present 4 design strategies derived from our study. Skaters' intentions can move between a focus on execution quality and a focus on trick originality any number of times within one skateboarding session. The strategies particularly aim to help designers support both execution quality-focused and originality-focused trick experiences in one integrated design, to allow skaters to seamlessly move between both intentions whenever desired.

\section{Capitalize on the Environment's Affordances for} Sharing of Trick Representations

Skateboarding involves interaction with the built environment that the athletes inhabit [3,11,13]. This environment affords sharing of trick representations, as it is often shared amongst many skaters. Skateboarders can see and hear each other's tricks and discuss their trick experiences easily. By incorporating trick representations into the environment, designers can capitalize on this affordance for sharing.

We found that sharing can support both execution qualityfocused and originality-focused trick experiences: in Copy Paste Skate the sharing of a skater's trick representation inspired others to either do a better trick execution than their fellow skater, or achieve aesthetically more pleasing lines by trying out unconventional tricks.

Many sports environments consist of mostly open space (e.g. figure-skating rings, snowboard parks, etc.), which allows for unobstructed perception of visual trick representations (e.g. trails on ice and snow) by people in the space. However, a challenge arises in sports where athletes move around large obstacles, corners, or even through different spaces (e.g. in parkour). Technology might offer unique opportunities for supporting the sharing in such sports, e.g. by displaying trick representations on one side of an obstacle or wall while the athlete is executing their trick on the other side.

This strategy aligns with previous work that suggests that integrating technology into a physical environment can make information presented through this technology more public [2]. Furthermore, this strategy feeds into the current trend of using technology to share exertion data between athletes [12]. We extend this work by suggesting that sharing can support both execution quality- and originalityfocused experiences, and that considering how the physical environment already supports sharing can be beneficial for designers.

\section{Allow for Persistent Traces of Temporal Tricks}

Skateboarding tricks are temporal, sometimes lasting less than a second. Technology allows for playing with this temporality, for example by offering representations of tricks that persist over time. In Copy Paste Skate the line remained visible after each trick attempt. This enabled skateboarders to compare their last trick with previous ones, allowing them to see progress, both in terms of executionquality and originality.

Previous work has already highlighted the value of using technology to enable lasting artistic creations through bodily exertion, suggesting it can help motivate users to engage in more physical activity [7]. We suggest that this persistent quality has additional value: it can support execution quality-focused as well as originality-focused trick experiences.

Tracks in the snow in snowboarding or dirt in freestyle motocross naturally offer traces. However, technology could augment these with additional or more detailed information, for example a measurement of the height of a jump.

\section{Carefully Consider the Level of Abstraction of Trick Representations}

Tricks often involve intricate board and body movements, which technology can represent in a realistic fashion (e.g. video; a popular medium amongst skaters). However, we recommend carefully weighing the advantages and disadvantages of both realistic and abstract trick representations when designing to support trick execution quality and originality. In Copy Paste Skate, the abstract visuals were lauded for helping skaters focus on improving specific trick aspects as well as for stimulating creativity. However, the absence of contextual information (e.g. the body) was missed when skaters desired to read into their execution in more detail (F6). To address this while preserving some of the qualities of abstract representation, an improved version of the system could for example feature a second line representing the movement trajectory of a point on the body.

Previous work highlights how abstract representations can support exertion performance [4] or creativity in exertion [19]. We build on this by proposing that supporting performance (i.e. execution quality) and supporting creativity are not mutually exclusive intentions, and that some sportspeople, like skateboarders, can use trick representations to support changing intentions throughout the activity.

\section{Offer Trick Representations of Strong Aesthetic Quality} It has been previously suggested that aesthetic information can be a reward for skateboarding tricks [7] and that aesthetic representations can support experimentation in sports [19], which relates to our originality-focus. In Copy Paste Skate, the projected line was characterized as having strong aesthetic qualities, with which the skateboarders actively engaged through their trick choices (F1,2). The interactive technology supported this aesthetic engagement by allowing skaters to examine the projected lines after each attempt, and hone them with every new attempt.

Some trick-focused sports revolve largely around intricate movements of the body alone (e.g. pirouettes performed in 
competitive diving or acrobatics). In such sports representations of these bodily movements might naturally possess strong aesthetic qualities. Sports where motions of other elements (such as the waves in surfing) also make up a large part of the aesthetics of the sport, incorporating additional variables (e.g. the shape of a wave) in representations might help translate aesthetic qualities of the sport to aesthetic representations. Another interesting avenue for future work is the challenge of achieving strong aesthetic qualities in aural or haptic feedback, which was found to be lacking in Copy Paste Skate (F1).

\section{CONCLUSIONS}

We have presented Copy Paste Skate, a novel interactive system for skateboarding that aims to support the experience of attempting tricks. By analyzing skateboarders' experiences with the system we identified a range of ways in which interactive technology can support the experience of skateboarding. This allowed us to reveal two key design dimensions that highlight how designing for skateboarding means supporting a focus on trick execution quality as well as a focus on the originality of tricks. Additionally, we presented four design strategies for how to support both dimensions in one integrated design. Although particularly useful for designers interested in creating interactive technologies for skateboarding, our work could also be of value for designers creating interactive technologies for other trick-based sports, such as freestyle snowboarding, BMX, freestyle motocross, parkour or acrobatics. Trick-focused sports that do not revolve around single isolated tricks (e.g. surfing, which we are interested in examining next) might require some of our strategies to be adapted.

In sum, we have shown how interactive technology can support various aspects of skateboarders' trick experience, and have provided concrete handles to help designers design for this. We hope others will build on this in their work by further exploring how interactive technology can support people in the exciting yet underexplored domain of trick-focused sports.

\section{ACKNOWLEDGEMENTS}

We would like to thank Eric Dittloff, Andrew Lewis, Chris Jones, Riverside Skatepark, and the CHI and TEI community.

\section{REFERENCES}

1. Anlauff, A., Weitnauer, E., Lehnhardt, A., Schirmer, S., Zehe, S., and Tonekaboni, K. A method for outdoor skateboarding video games. ACE'10, (2010), 40-44.

2. Bimber, O. and Raskar, R. Spatial Augmented Reality: Merging Real and Virtual Worlds. A K Peters, Ltd., Wellesly, 2005.
3. Borden, I. Skateboarding, Space and the City: Architecture and the Body. Berg, New York, USA, 2001.

4. Broker, J.P., and Crawley, J.D. Advanced Sport Technologies: Enhancing Olympic Performance. 19 ISBS, (2001), 323-327.

5. Buttkicker. thebuttkicker.com/lfe-kit.php.

6. Eness. The Making of a Light Session. vimeo.com/18525296.

7. Fan, C., Forlizzi, J., and Dey, A.K. A Spark Of Activity: Exploring Informative Art As Visualization For Physical Activity. UbiComp'12, (2012), 2-5.

8. Langlotz, T., Zingerle, M., Grasset, R., and Kaufmann, H. AR Record \& Replay: Situated Compositing of Video Content in Mobile Augmented Reality. OZCHI'12, (2012), 318-326.

9. Marc, B., Kilian, F., and Tr, G. SwimMaster : A Wearable Assistant for Swimmer. UbiComp'09, (2009), 215-224.

10. Michalko, M. Thinkertoys: A Handbook of CreativeThinking Techniques. Ten Speed Press, 2006.

11. Németh, J. Conflict, Exclusion, Relocation: Skateboarding and Public Space. Journal of Urban Design 3, 11 (2006), 311-327.

12. Nike. Nike+. nikeplus.nike.com/plus/.

13. Nolan, N. The ins and outs of skateboarding and transgression in public space in Newcastle, Australia. Australian Geographer 34, 3 (2003), 311-327.

14. Otoplasma. Skate Sonic. otoplasma.com/skatesonic. 15. Pijnappel, S. and Mueller, F. 4 Design Themes for Skateboarding. CHI'13, ACM Press (2013).

16. Reynell, E. and Thinyane, H. Hardware and software for skateboard trick visualisation on a mobile phone. SAICSIT'12, (2012), 253-261.

17. Rigby, S. and Ryan, R. Glued to Games: How Video Games Draw Us in and Hold Us Spellbound. Praeger Publishers, Westport, 2011.

18. Seifert, T. and Hedderson, C. Intrinsic Motivation and Flow in Skateboarding: An Ethnographic Study. Journal of Happiness Studies 11, 3 (2009), 277-292.

19. Sheridan, J.G., Bayliss, A., Bryan-kinns, N., and Mary, Q. iPoi : acceleration as a medium for digital live art. UbiComp '06, (2006).

20. Skataviz. design-io.com/projects/Skataviz/.

21. Stienstra, J., Overbeeke, K., and Wensveen, S. Embodying Complexity Through Movement Sonification: Case Study on Empowering the Speedskater. CHI'11, ACM Press (2011), 39-44.

22. Tholander, J. and Johansson, C. Design qualities for Whole Body Interaction - Learning from Golf, Skateboarding and BodyBugging. NordiCHI 2010, ACM Press (2010), 493-502.

23. Wikipedia. Tony Hawk's Pro Skater. en.wikipedia.org/wiki/Tony_Hawk's_Pro_Skater. 